\title{
Wer sind eigentlich diese Gamer?
}

Ich möchte diesen Teil des Buches mit einer einfachen Übung beginnen. Bitte schließen Sie die Augen und versuchen Sie sich, einen Gamer vorzustellen. Dann nehmen Sie ein Blatt Papier in die Hand und schreiben bitte die fünf wichtigsten Punkte auf, die Ihrer Meinung nach einen Gamer am besten beschreiben. Keine Sorge, es gibt bei dieser Aufgabe kein richtig oder falsch. Aber ich lasse Teilnehmer in meinen Workshops diese Aufgabe machen, und ich stelle fest, dass das Bild, welches die meisten Menschen von Gamern haben, sehr interessant ist. In den meisten Fällen werden Gamer als männliche Teenager beschrieben. Manche Menschen glauben, dass Gamer selten andere Hobbys haben und manchmal ein bisschen ungepflegt sind. Lauter nette kleine Stereotypen. Aber nun möchte ich Sie mit jemandem bekannt machen, der ebenfalls Gamer ist, aber wahrscheinlich so gar nicht in das typische Muster passt: Mich. Christoph Deeg. Gamertag: „crocksberlin“.

Ich selber bin Gamer. In dem Moment, in dem ich dieses Buch schreibe, bin ich 39 Jahre alt. Ich habe sehr früh mit Gaming angefangen. Schon auf den ersten Atari-Konsolen habe ich Pixel und vieles andere gejagt. Ich selber hatte keine dieser Konsolen. Aber in meinem Freundeskreis gab es einige Jungs, die eine Atari-Konsole hatten. Und die Zimmer oder Partykeller dieser Freunde waren heilige Orte. Hier trafen wir uns, um zu spielen. Und meistens ging es darum zuzugucken. Wirklich spielen konnten immer nur ein oder zwei Spieler. Die anderen mussten zuschauen. Gaming war ein soziales Erlebnis. Unsere Eltern versorgten uns mit belegten Brötchen und Getränken, und sie waren so nett, uns beim Spielen nicht weiter zu stören. Wir konnten also in Ruhe unserem Hobby nachgehen. Und wir hatten jede Menge Spaß dabei. Nun waren die Computerspiele bei weitem nicht das einzige, was uns verband. Wir spielten draußen Fußball, wir bastelten Baumhäuser und Flöße, und wir wanderten durch unser Dorf. Aber Gaming war ebenso Teil unserer Lebensrealität wie alles andere, was wir so taten. Unsere Eltern konnten sich das nicht erklären. Was sollte daran spannend sein, mit solchen pixeligen Bildern und piepsigen Geräuschen zu spielen? Warum waren alle anderen bereit, daneben zu sitzen und zu warten, bis sie dran waren? Zudem war es für diejenigen von uns, die keine eigene Konsole zu Hause hatten, problematisch, gegen die „Profis“, die täglich üben konnten, zu bestehen. Wir versuchten unser Bestes. Aber in der Regel war meine Spielzeit mehr als limitiert.

Dann eines Tages hatten wir bei uns im Dorf so eine Messe. Es war direkt am Fluss, dort wo sonst im Sommer immer die Kirmes stattfand. Nun standen dort also sehr große Zelte und sehr viele Menschen und Unternehmen präsentierten ihre Ware und Dienstleistungen. Dieser Ort war Langeweile pur. Es gab nichts für uns Teenager. Wir verstanden nichts von den ganzen Baumaschinen, Heizungsventilen, Reinigungssprays, Messer etc. Doch an einem Stand fand Magie statt: Dort stand ein kleiner Computer. Ich glaube, es war ein C64, und auf diesem Computer konnten wir Spiele spielen. Es war mein erster Kontakt mit einem Computer, und dieses Spiel gab mir die Möglichkeit, mit dieser Maschine zu arbeiten. Ich beherrschte keine Programmiersprache, ich wusste bei einigen der Tasten nicht einmal, was sie bedeuteten. Und doch saß ich nun wie ein Profi vor diesem Gerät und spielte. Irgendwann wollte ich ein anderes Spiel spielen, und ich musste lernen, wie man neue Spiele lädt. Ich kann mich nicht mehr an den Vertreter der Computerfirma erinnern. Aber ich weiß, dass dies mein erster Kontakt mit Computern war. Und in diesem Moment lernte ich, dass so ein Computer Spaß machen kann.

Bis dato hatte ich eigentlich kein Interesse an diesen Geräten. Ich war eher musisch begabt - was u.a. dazu führte, dass ich später Musik studierte und Mathe und Physik nur in Ansätzen verstanden habe. Das Spiel ermöglichte mir den Zugang zu 
einer neuen Hochtechnologie. Aber das war nur der Beginn der Reise. Ich kaufte mir keinen C64, aber in meinem Freundeskreis gab es immer mehr dieser Geräte. Und plötzlich konnte man überall die Kopien der Spiele bekommen. Letztlich waren das alles ziemlich einfache Spiele. Im Vergleich zu heutigen Games war das alles eher schwach. Aber damals war es die große weite Welt.

Irgendwann hatte ich dann in der neunten Klasse ein Berufspraktikum zu machen. Ich hatte keine große Lust darauf, denn ich wollte studieren und keine Berufsausbildung beginnen. Aber da es nun mal sein musste, suchte ich mir etwas im Umfeld meines Dorfes. Dort gab es u. a. ein großes Pharma-Unternehmen und einige meiner Freunde hatten gute Kontakte zu den einzelnen Abteilungsleitern. Und so begann ich mein Praktikum im Bereich „Technisches Zeichnen“. Ich möchte es kurz machen: Ich verfüge mit Sicherheit über einige Kreativität und viele tolle künstlerische Ansätze. Als technischer Zeichner war und bin ich eine Niete. Aber ich musste nun ein paar Wochen da bleiben und sogar noch einen Bericht schreiben. Ich musste mich also irgendwie mit der Situation arrangieren. Es dauerte nicht lange und es zeichnete sich eine Lösung ab. Ich hatte zwar keine Ahnung von Technischem Zeichnen, aber ich konnte Skat spielen, und mein „Chef“ spielte jede Pause Skat. Leider war einer der Spieler ausgefallen. Also wurde ich quasi Auswechselspieler. Wenn man aus mir schon keinen technischen Zeichner machen konnte, war ich wenigstens als Skatspieler zu gebrauchen. Ansonsten hatte ich mehr oder weniger frei. Also begann ich mich überall in den Büros umzusehen, und dann fand ich die Lösung für mein Problem: In einem Büro stand ein Computer mit einem einfachen Computerspiel. Somit war meine Zeit in dem Unternehmen nicht umsonst. Ich musste lernen, wie man den Computer bzw. das Spiel aktiviert, ich musste das Spiel lernen und ich musste spielen. Und natürlich musste ich pünktlich zum Skatspielen erscheinen. Alles in allem eine gute Zeit. Und man half mir sogar beim Abschlussbericht.

Wenig später kam die nächste Herausforderung: Mein kleiner Bruder hatte ein Nintendo NES nach Hause gebracht und nun wurde Super Mario Teil meines Lebens. Leider war ich in der ersten Zeit zum Zuschauen verdammt. Mein Bruder war einfach um Klassen besser als ich. Er konnte stundenlang vor dem System sitzen und neuen Lösungswege finden. Es war beeindruckend und natürlich zeigte er mir alle neuen Errungenschaften. Auf jeden Fall hörte ich nun viele Nachmittage die Mario Bros Melodie. Und meine Gaming-Reise ging weiter.

Ein paar Jahre später war ich u.a. dabei, politisch aktiv zu sein. Wenn man fünfzehn Jahre alt ist, probiert man gerne mal aus, wie es ist, Politik zu machen. Ich hatte einen guten Freund - der im Gegensatz zu mir wirklich eine echte politische Karriere hingelegt hat -, und wir beide waren nun beinahe jeden Tag zusammen und bastelten an der demokratisch legitimierten Weltherrschaft :-) Er war ein bisschen älter als ich und hatte einen PC, genauer gesagt einen sogenannten 286er. Das war ein Computer, auf dem schon Windows laufen konnte. Aber letztlich nutzte man nur DOS und den Norton Commander. Mal wieder wusste ich nicht so richtig, was man mit diesem Gerät Sinnvolles anfangen sollte, bis wir „North and South“ spielten. Dieses Spiel behandelte den amerikanischen Unabhängigkeitskrieg. Es war mit Sicherheit keine historische Darstellung. Der Unabhängigkeitskrieg war nur die Bühne. Man hatte ein paar Einheiten und versuchte, damit den Gegner zu besiegen. Wir spielten das Spiel zusammen gegeneinander und schon versuchte ich, neue Wege der Eroberung zu finden. Das Spiel war weder besonders komplex noch anspruchsvoll. Also verloren wir irgendwann die Lust. Und dann kam „Railroad Tycoon“ und wenig später sogar „Civilisation“. Und ab da wurde es groß. Diese Spiele waren für damalige Verhältnisse komplexe Wirtschafts- und Gesellschaftssimulationen. Hier musste man wirklich lernen und ausprobieren. Wir tauschten uns mit anderen Spielern aus. Wir entwickelten 
Strategien. Wir lernten. Und diese beiden Spiele waren der Grund, warum ich nun auch einen eigenen Computer brauchte. Also suchte ich mir mal wieder einen Ferienjob. Das Geld des letzten Jobs hatte ich für mein Schlagzeug ausgegeben. Ich bekam irgendwann meinen Computer und dann konnte ich endlich spielen. Ich fühlte mich wie der Gott der Hochtechnologie. Ich hatte einen eigenen Computer. Natürlich hatte ich meiner Mutter gesagt, dass ich mit dem Gerät lernen wollte und rückwirkend betrachtet, habe ich das auch. Aber ehrlich gesagt, ging es nur um das Zocken.

Damit war meine Gamingreise aber noch nicht zu Ende. Ein paar Jahre später zog ich nach Bremen um zu studieren. Zu dieser Zeit begann ich intensiv, Musik zu machen, und schließlich hatte ich nicht nur eine Band sondern vor allem einen guten Freund, mit dem ich Tag und Nacht Musik hörte, spielte und komponierte. Zur damaligen Zeit nutzten wir einen Atari 1064ST, um mit einem Programm namens CUBASE Musik zu komponieren. Der Computer war dabei die Zentrale eines Midistudios. Über eine sogenannte Midi-Schnittstelle haben wir verschiedene Synthesizer durch den Computer ansteuern können. Wir gaben also Noten in den Computer ein und legten gleichzeitig fest, wie diese Noten von welchem Synthesizer auf welche Art und Weise wiedergegeben wurden. Das Ganze wurde im Tonstudio auf großen Mehrkanal-Recordingsystemen aufgenommen und dann abgemischt. Diese Arbeit hatte gleich doppelt etwas mit Gaming zu tun. Zum Einen bin ich mir sicher, dass ich die damalige Musiksoftware und -hardware nicht hätte begreifen und/oder nutzen können, wenn ich nicht vorab durch die Games erste Erfahrungen auf dem Computer gesammelt hätte. Die Games hatten mir die Tür in die digitale Welt eröffnet. Zum Anderen bestand die Arbeit im Tonstudio kontinuierlich aus Try and Fail. Ich weiß nicht, wie viele Tracks wir aufgenommen haben. Wir verbrachten Wochen in diesem Studio und probierten unglaubliche Mengen an Sounds und Geräuschen, an Melodien und Geräten aus. Fehler machen bedeutete nie etwas Schlechtes. Wir lernten mit jedem Misserfolg weiter, und ohne diese Zeit hätte ich mit Sicherheit niemals die Aufnahmeprüfung an der Musikhochschule geschafft und das Musikstudium auch niemals erfolgreich abgeschlossen.

Auch während des Studiums war Gaming Bestandteil meiner Lebensrealität. Wenn wir mit Bands im Tonstudio arbeiteten, gehörte eine XBOX oder eine Playstation immer mit dazu. Wenn man sich intensiv mit Musik beschäftigte, brauchte man etwas zur Entspannung. Auch wenn man es nicht glauben mag, aber die Arbeit im Tonstudio bzw. das Ausarbeiten neuer Songs ist sowohl physisch als auch psychisch sehr anstrengend. Mit den Spielkonsolen konnten wir uns eine kleine Auszeit nehmen und gegeneinander spielen. Gleichzeitig hatte ich meine erste Retro-GamingErfahrung. Es war eines dieser Wochenenden, bei denen man nach Hause in sein Dorf fährt und seine Familie und alte Freunde besucht. Einer meiner Freunde hatte seinen alten C64 reanimiert und hatte das Spiel „Vermeer“ gefunden. Dieses Spiel ist ein rundenbasiertes einfaches Strategiespiel, welches letztlich nur aus Text mit minimaler Grafik besteht. Man spielt gegeneinander und baut ein internationales Handelsimperium auf. Die Grafik ist schwach und das Gameplay einfach - und wir hatten ein ganzes Wochenende einen unglaublich großen Spaß. Am Ende suchte ich auf dem Dachboden wieder meine alten Gamingsysteme heraus und begann mich intensiver mit Retro-Gaming zu befassen.

Wieder einige Jahre später wohnte ich für ein Jahr mit meinem Bruder zusammen in Berlin. Er hatte uns eine XBOX gekauft, und wir hatten unsere Liebe für den Shooter „Halo“ entdeckt. Nächtelang saßen wir vor dem Fernseher und jagten gemeinsam Aliens. Wenn wir davon genug hatten, spielten wir gegeneinander „Ghost-Recon“. Natürlich haben wir in dieser Zeit auch andere Dinge getan, aber Gaming war etwas, was uns mehr verband als vieles andere. 
Warum habe ich Ihnen diese Geschichte aufgeschrieben? Wenn Sie den Text genau durchlesen, dann können Sie sehr viel über das Thema Gaming lernen. Da sind die Gaming-Erlebnisse eines beinahe 40 Jahre alten Mannes, der auf eine lange individuelle Gaming-Historie zurückblicken kann. Und damit bin ich nicht allein. Ich gehöre vielmehr zu einer Generation, die bereits mit Gaming aufgewachsen ist.

Gaming war bei mir immer eine soziale Aktivität. Gaming war etwas, was in der Gruppe passierte. In den Anfangsjahren ließen die Technologien ein „alleine Spielen“ gar nicht zu. Gaming fand im Freundeskreis statt und es war Teil meiner Lebensrealität wie viele andere Aktivitäten auch. Unsere Eltern schufen Räume bzw. Plattformen, auf denen wir dann unserem Hobby nachgehen konnten. Nicht die Schule, sondern Gaming sorgte bei uns für erste Erfahrungen mit modernen Kommunikations- und Medientechnologien. Natürlich sorgte man für eine Balance, d.h. wir spielten nicht nur Computerspiele. Wir waren ebenso in Sportvereinen etc. aktiv. Rückblickend finde ich es besonders interessant, dass mir damals wie heute das Spielen zusammen mit anderen weitaus größeren Spaß macht, als das Spielen alleine.

Natürlich gibt es auch Menschen in meinem Alter, die keine so intensive Verbindung zu Games haben. Und ich möchte auch auf keinen Fall behaupten, dass Gaming besser ist als beispielsweise ein Buch zu lesen. Aber Gaming ist eben auch nicht schlechter als das Lesen von Büchern. Und ja, ich spiele Computergames, und ich lese gerne Bücher, und ich gehe gerne spazieren etc.

Gaming kann nicht nur einen Einfluss auf einzelne Menschen haben. Es kann ebenso ganze Bevölkerungsschichten verändern bzw. bewegen. Einen solchen Effekt können wir z. B. in Südkorea beobachten. Dort wurde bereits Mitte der 90er Jahre mit der Entwicklung und Realisierung einer landesweiten Strategie für die Digitalisierung der Gesellschaft begonnen. Neben dem massiven Ausbau der digitalen Infrastruktur und einem Schulungsprogramm für 20\% der Gesamtbevölkerung wurde Gaming zu einem Motor der gesellschaftlichen Entwicklung. In kurzer Zeit wurden über 20.000 Internet-Cafes eröffnet. Und diese Cafes entwickelten sich zu neuen sozialen Orten. In diesen Cafes begannen immer mehr Südkoreaner Spiele wie z. B. „Starcraft“ zu spielen. Es dauerte nicht lange, und es entwickelte sich eine regelrechte Gaming-Kultur. Die Regierung Südkoreas sah, welchen positiven Einfluss Gaming auf die Digitalisierung der Gesellschaft hatte. Aus diesem Grund wurde Gaming nicht etwa als Gefahr, sondern vielmehr als Chance gesehen. Es wurde entschieden, Gaming aktiv zu unterstützen und so hat Gaming einen großen Anteil zur erfolgreichen Digitalisierung der Gesellschaft Südkoreas beigetragen.

Wenn wir uns die Daten zu Gamern anschauen, dann stellen wir fest, dass Gaming ein gesamtgesellschaftliches Phänomen geworden ist. Der Bundesverband Interaktiver Unterhaltungsindustrie (BIU) hat dazu Zahlen veröffentlicht ${ }^{4}$ :

1. Mehr als $25 \%$ der deutschen Bevölkerung sind aktive Gamer

2. Der Frauenanteil beträgt $50 \%$

3. Das Durchschnittsalter beträgt 32 Jahre

4. In allen Bildungsschichten lassen sich Gamer finden

5. In 50\% aller Familien mit Kindern unter 18 Jahren wird gespielt

6. In allen Einkommensklassen wird gespielt, wobei der Anteil der Gamer in den höheren Einkommensklassen steigt

4 Quelle: http://www.biu-online.de/de/fakten/gamer-statistiken.html, Datum der letzten Abfrage: 14.07.2013 
Das bedeutet, dass Bibliotheken das Thema Gaming nicht nur als Thema für männliche Jugendliche ansehen sollten. Vielmehr lassen sich damit Menschen in allen Altersklassen und allen Einkommens- und Bildungsschichten erreichen.

Es sollte ebenso nicht vergessen werden, dass es eine große Zahl an Erwachsenen gibt, die eine eigene Gaming-Vergangenheit haben. Hier ist eine eigene Kultur entstanden, welche man ohne weiteres mit der Buchkultur vergleichen kann. Während Bibliotheken nicht nur Bücher anbieten, sondern auch Teil der Kultur rund um das Buch geworden sind bzw. dies auch aktiv kommunizieren, ist dies im Bereich Gaming selten der Fall. Damit Sie mich nicht falsch verstehen: es geht nicht darum, die Bibliothek zu einer Spielhölle werden zu lassen. Es geht auch nicht um einen Konkurrenzkampf zwischen Gaming und beispielsweise Büchern. Aber wenn wir uns die Zahlen ansehen, dann müssen wir akzeptieren, dass Gaming für nahezu alle Gruppen unserer Gesellschaft interessant sein kann. Natürlich gibt es Unterschiede, z. B. hinsichtlich der Präferenzen bezüglich der gespielten Spiele, und natürlich erleben wir z.B. auf der Gamescom nur einen Ausschnitt aus dieser Gaming-Kultur. Aber die Zahlen zeigen, dass Gaming kein Nischenthema ist.

Recherchieren Sie im Internet oder in der analogen Welt, ob es in Ihrem geographischen Umfeld eine Gaming-Community gibt. In manchen Fällen sind diese Communities gerne bereit, Sie zu unterstützen.

Fragen Sie in Ihrem Team nach, wer von den Kollegen Gamer ist oder aber Kinder hat, die spielen. Diese Personen können Ihnen helfen. Vor allem die Kinder sind tolle Ideengeber. Schaffen Sie Ihnen einen Kreativraum. Machen Sie sie zu „Gaming-Beratern“. Auf diese Art und Weise werden Ihre ersten Schritte im Bereich Gaming bereits ein eigenes Projekt. Wenn Sie wollen, können Sie einen GamingBeirat gründen, der Sie beraten soll. Dazu später mehr. 